\title{
La odontología pediátrica en el siglo XXI
}

Noemí Bordoni 1,a

${ }^{1}$ Universidad de Buenos Aires, Instituto de Investigaciones en Salud Pública. Buenos Aires, Argentina.

a Doctora en Odontología.

\section{Correspondencia:}

Noemí Bordoni

Correo electrónico: nbordoni14@gmail.com

Universidad de Buenos Aires. Facultad de Odontología. Marcelo T.de Alvear

$2142,5^{\circ}$ piso, Sector B, 1122, Buenos Aires, Argentina.

Recibido: 22/03/19

Aceptado: $26 / 03 / 19$

Publicado: 05/06/19

\section{Pediatric dentistry on the twenty first century}

El mundo se desestructura y reestructura permanentemente. Los procesos de interacción entre actividades humanas se cruzan en el rango de esferas tales como la económica, la social y la cultural, generando cambios en las dimensiones espaciales, temporales y cognitivas. La dimensión espacial es percibida hoy en un espacio físico reducido por las interacciones humanas más frecuentes y tecnológicamente sustentadas. Esa permanente comunicación tecnológica abre caminos para los contactos pero estrecha la posibilidad de los encuentros. La dimensión temporal plantea también un acortamiento del tiempo real y del percibido en el que se desenvuelven esas interacciones. La dimensión cognitiva (conjunto de ideas, culturas, creencias y valores de las personas) se arriesga a las tensiones generadas, por un lado, por el predominio de la razón instrumental y la valoración del conocimiento objetivo mientras que, por otro, se revalorizan los valores de la subjetividad y su comprensión desde lo interdisciplinar.

Frente a este mundo: ¿es pertinente la formación de especialistas desde la perspectiva tradicional? ¿Es suficiente conocer las técnicas y los materiales dentales que frecuentemente se cuestionan y descartan aún antes que los profesionales alcancen la automatización requerida para su uso como expertos? Los nuevos escenarios redefinen los perfiles del grado y de los posgrados profesionales. Las diferentes categorías que ofrecen las instituciones de educación superior deben formar profesionales con la capacidad para interpelarse y analizar críticamente sus prácticas a partir de la evidencia científica contextualizada, abordando las esferas sociales, culturales y económicas que le permitan comprender la complejidad y la multi-referencialidad requeridas para alcanzar la efectividad en sus funciones.

Un ejemplo revelador es la transición conceptual que acompañó a la atención odontológica de la niñez y la adolescencia. Hace tiempo que se ha reconocido que el saber odontopediátrico no se expresa únicamente en las competencias del "saber hacer" en la clínica. El saber odontopediátrico es un espacio en construcción permanente que exige competencias para:

- seguir la movilidad del pensamiento de los actores en el campo - niño/niña, padre/madre y profesional - y las relaciones intersubjetivas que van elaborando,

- identificar nuevas lecturas e interpretaciones para los viejos problemas, para los problemas emergentes y para su reconocimiento causal, sostenido tanto por el soporte tecnológico, como por su contrastación cuanti-cualitativa,

- participar o dirigir investigaciones tendientes a las resoluciones de los nuevos problemas de salud o a la toma de decisiones para su sensata aplicación, 
- dirigir estratégicamente un servicio de odontopediatría o el componente odontológico de un programa de salud integral, incluyendo su gestión económico-financiera,

- fortalecer el impacto positivo producido en los diferentes momentos del curso de vida mediante una temprana promoción de la salud y por el control de los factores de riesgo, y

- articularse con otros dominios para la resolución de problemas que, por su complejidad, requieran el apoyo de otros campos disciplinares.

Esto es lo que exige el mundo de hoy y son las instituciones universitarias las que deben estimular el lujo de la duda científica, galvanizar la formulación de preguntas y poner a prueba las respuestas para no canonizar verdades provisoriamente demostradas. Sus equipos deben proveer de significado y sentido a la institución, porque los grupos humanos no operan en el vacío: son contenidos, conformados y parametrados por el escenario donde se desenvuelve su quehacer. Las universidades reclaman ese protagonismo e instalan en sus agendas la preocupación por generar profesionales con la capacidad de reflexión necesaria para encarar éticamente la construcción de sus conocimientos, validarlos y transferirlos a toma de decisiones con impacto positivo sobre la salud.

De allí, la fuerza que adquiere la extensión universitaria entendida como expresión pragmática de la vinculación universidad-contexto social, resultante de la interacción entre la sociedad con sus múltiples expresiones y demandas para la solución de problemas concretos y las universidades, con sus formas de producción del conocimiento y las nuevas formas de organizarlos, distribuirlos e intercambiarlos en los diversos escenarios del equipamiento social ${ }^{1}$.

¡Ese es su tiempo!

\section{Referencias bibliográficas}

1. Bordoni N. La extensión como objeto de investigación. Análisis desde el campo de la salud. +E: Revista de extensión universitaria. 2018;8(9):126-144. DOI:10.14409/ extensión .v8i9.jul.dic.8951. 